\title{
Maximum Theoretical Efficiency Limit of Photovoltaic Devices: Effect of Band Structure on Excited State Entropy
}

\author{
Frank E. Osterloh* \\ Department of Chemistry, University of California-Davis, One Shields Avenue, Davis, California 95616, United States
}

ABSTRACT: The Shockley-Queisser analysis provides a theoretical limit for the maximum energy conversion efficiency of single junction photovoltaic cells. But besides the semiconductor bandgap no other semiconductor properties are considered in the analysis. Here, we show that the maximum conversion efficiency is limited further by the excited state entropy of the semiconductors. The entropy loss can be estimated with the modified Sackur-Tetrode equation as a function of the curvature of the bands, the degeneracy of states near the band edges, the illumination intensity, the temperature, and the band gap. The application of the second law of thermodynamics to semiconductors provides a simple explanation for the observed high performance of group IV, III-V, and II-VI materials with strong covalent bonding and for the lower efficiency of transition metal oxides containing weakly interacting metal $\mathrm{d}$ orbitals. The model also predicts

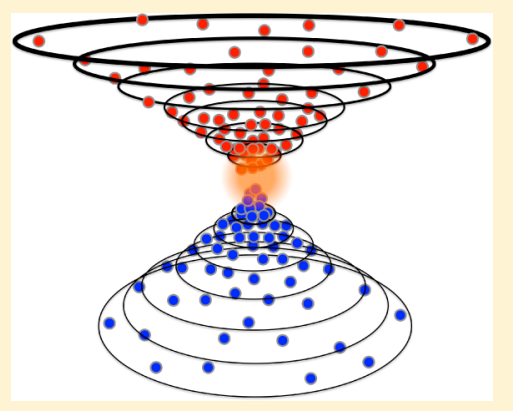
efficient energy conversion with quantum confined and molecular structures in the presence of a light harvesting mechanism.

SECTION: Energy Conversion and Storage; Energy and Charge Transport

$S^{e}$ emiconductors are at the heart of solar cells and photoelectrochemical devices for solar energy conversion. The maximum theoretical efficiency of single junction devices can be estimated with the Shockley Queisser model, ${ }^{1}$ which calculates the ideal performance limit on the basis of the semiconductor band gap and the energy of the incident blackbody radiation (see also Ross et al. ${ }^{2,3}$ ). Besides the band gap, no other semiconductor properties are considered in the model. This cannot account for the efficiency variations observed for real photovoltaic devices today. ${ }^{4-6}$ Here, we show that the extractable work from excitonic semiconductors is further limited by entropy losses that are associated with the dilution of the photoexcited charge carriers in the semiconductor bands. These losses depend sensitively on the electronic structure of the semiconductor, that is, they are a materials property. They can be calculated with the SackurTetrode equation from the effective mass of the charge carriers. Low losses are predicted for semiconductors with strong covalent bonding and few atomic states, which provides a straightforward explanation for the high performance of single junction solar cells made from semiconductors in the covalent zincblende structure type $(\mathrm{Si}, \mathrm{GaAs}, \mathrm{CdTe}, \mathrm{CIGS})^{6}$ and for the recently discovered lead perovskites. ${ }^{7}$ The low effective density of states of these materials ensures concentration of charge carriers near the band edges. The entropy model also explains the low photon conversion efficiency of $3 \mathrm{~d}$ and $4 \mathrm{~d}$ transition metal oxides $\left(\mathrm{Fe}_{2} \mathrm{O}_{3}, \mathrm{TiO}_{2}, \mathrm{WO}_{3}\right)$ and sulfides, whose lower performance has traditionally been attributed to the lower mobility of the charge carriers in these materials. ${ }^{8-10}$ Lastly, the entropy model predicts high energy conversion efficiency for isolated quantum confined and molecular structures under illumination with concentrated light.

In 1980, Peter Würfel used the ideal gas model to describe the excited state energetics of a semiconductor and to calculate the maximum efficiency limit of a photovoltaic device. ${ }^{11,12}$ Light excitation produces electrons and holes, which relax on the submillisecond time scale to reach thermal equilibrium with the semiconductor lattice, and to form an ideal gas (Figure 1). Each conduction band electron (hole) in this gas then has the potential energy $E_{\mathrm{CB}}\left(E_{\mathrm{VB}}\right)$, kinetic energy $3 / 2 k T$, and volume energy $k T(=p v)$.

The free energy $\mu_{\mathrm{e} / \mathrm{h}}$ of an electron (hole) is less than the sum of these terms, because each charge carrier also has the entropy $\sigma_{\mathrm{e} / \mathrm{h}}$ (see eq 1 for an electron).

$$
\mu_{\mathrm{e}}=h_{\mathrm{e}}-T \sigma_{\mathrm{e}}=E_{\mathrm{CB}}+\frac{5}{2} k T-T \sigma_{\mathrm{e}}
$$

The entropy arises from the ability of the charge carriers to occupy several of many possible kinetic energy states in the conduction and valence bands. For an uncharged ideal gas particle, the entropy is given by the Sackur-Tetrode (ST) equation. For electrons/holes in a semiconductor, the equation is modified (eq 2) to account for the fact that electrons (and holes) have two spin orientations, which doubles the number of possible states.

$$
\sigma=\frac{5}{2} k+k \ln \left[\frac{2}{n}\left(\frac{2 \pi m^{*} k T}{h^{2}}\right)^{3 / 2}\right]
$$

The semiconductor lattice further modifies the entropy because it imposes restrictions on the motion of the charge carriers and the number of allowed kinetic states. This is illustrated in Figure 2 for three different model semiconductors.

Received: August 17, 2014

Accepted: September 8, 2014 


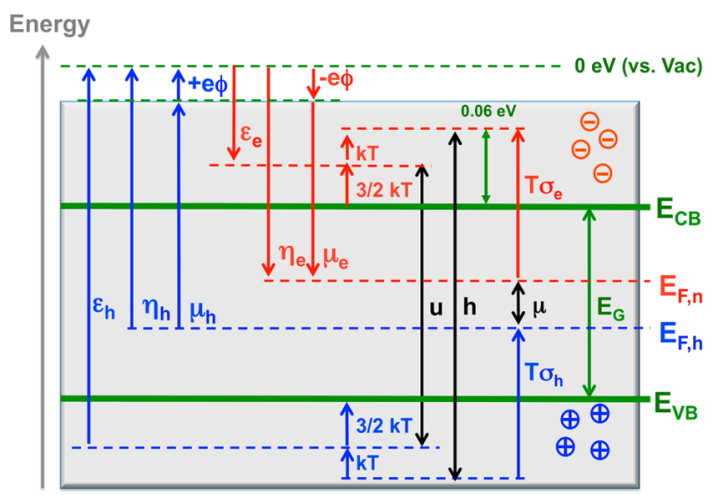

Figure 1. Physical and thermodynamic parameters for excited semiconductor in thermal equilibrium modified from Würfel. ${ }^{11,12}$ See also Salvador. ${ }^{13} \eta_{\mathrm{e}, \mathrm{h}}$, Electrochemical potentials of electrons and holes (e.g., as measured by photoelectrochemistry); $E_{\mathrm{F}, \mathrm{e} / \mathrm{h}}$, quasi-Fermi energies of electrons (equals $\eta_{\mathrm{e}}$ ) and holes (equals $-\eta_{\mathrm{h}}$ ); e $\phi_{\mathrm{e}, \mathrm{h}}$, electric potentials of electrons (negative) and holes (positive); $\mu_{\mathrm{e}, \mathrm{h}}$, chemical potentials of electrons and holes; $\varepsilon_{\mathrm{e} / \mathrm{b}}$, energy of electrons and holes (calculated from potential energy, kinetic energy, and electrostatic energy); $u$, internal energy of electrons-hole pair $\left(\varepsilon_{\mathrm{e}}+\varepsilon_{\mathrm{h}}\right) ; h$, enthalpy of electron/hole pair (includes volume energy); $E_{\mathrm{CB}} / E_{\mathrm{VB}}$, energy of conduction and valence band edges; $E_{\mathrm{G}}$, band gap energy; $T \Delta \sigma_{\mathrm{e} / \mathrm{h}}$, entropic energy of electrons or holes; $\mu$, chemical potential of electron-hole pair $\left(\mu=\mu_{\mathrm{e}}+\mu_{\mathrm{h}}=E_{\mathrm{F}, \mathrm{e}}-E_{\mathrm{F}, \mathrm{h}}\right)$ equals maximum extractable free energy.

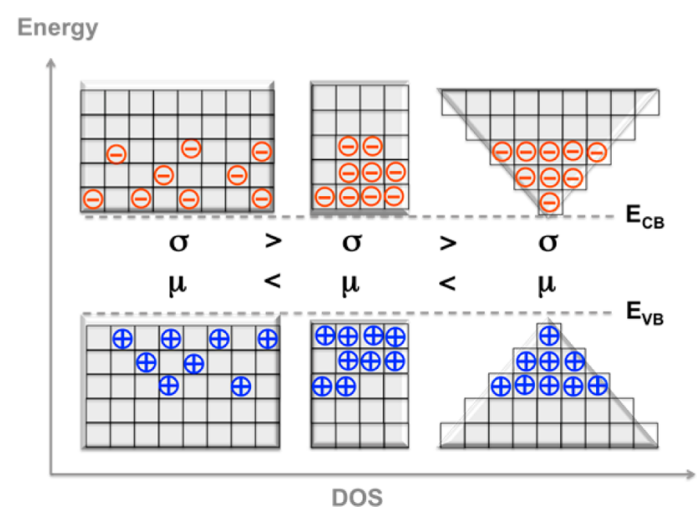

Figure 2. Dependence of free energy of excited semiconductors on the shape of the DOS near the band edges. Each square represents an allowed state. Only energy states within $2 k T(0.06 \mathrm{eV}$ at room temperature) of the band edges are occupied.

As can be seen, the number of effective states $N_{\text {eff }}$ (three rows of squares near band edges) that are available for electrons and holes depends on the density of state (DOS) distribution near the valence and conduction band edges. The larger the DOS, the more states are available near the bottom, and the greater $N_{\text {eff. }}$ For the model systems in Figure 2, the entropy per charge carrier can then be estimated with the Boltzmann formula $\sigma=$ $1 / n \times k \times \ln W_{\text {Tot }}$. The formula is not exact because of the small number of states in the example. Here, $W_{\text {tot }}$ gives the possible number of combinations to distribute $n=9$ electrons over $N_{\text {eff }}$ states. For $N_{\text {eff }}=24,12,9, W_{\text {tot }}=24 ! /[(24-9) ! 9 !]$, $12 ! /[(12-9) ! 9 !], 9 ! /[(9-9) ! 9 !]$, and $\sigma=2.2 \times 10^{-23}, 8.3 \times$ $10^{-23}, 0.0 \mathrm{~J} / \mathrm{K}$. This shows that the entropy-related energy loss $\mathrm{T} \sigma$ decreases from left to right. The free energy $\mu=h-T \sigma$ increases in the same direction, and so does the maximum electric power that can be extracted from these model semiconductors.
For real semiconductors the entropy of electrons and holes can be calculated with the modified ST equation, using the effective masses of electrons and holes. That is possible because in solid-state physics the effective mass of the electrons/holes in a semiconductor is related to the curvature of the energy-DOS plot and to the effective density of states. For a free electron moving in vacuum, the kinetic energy scales with its squared momentum. The corresponding $E / k$ curve (Figure 3 ) is a
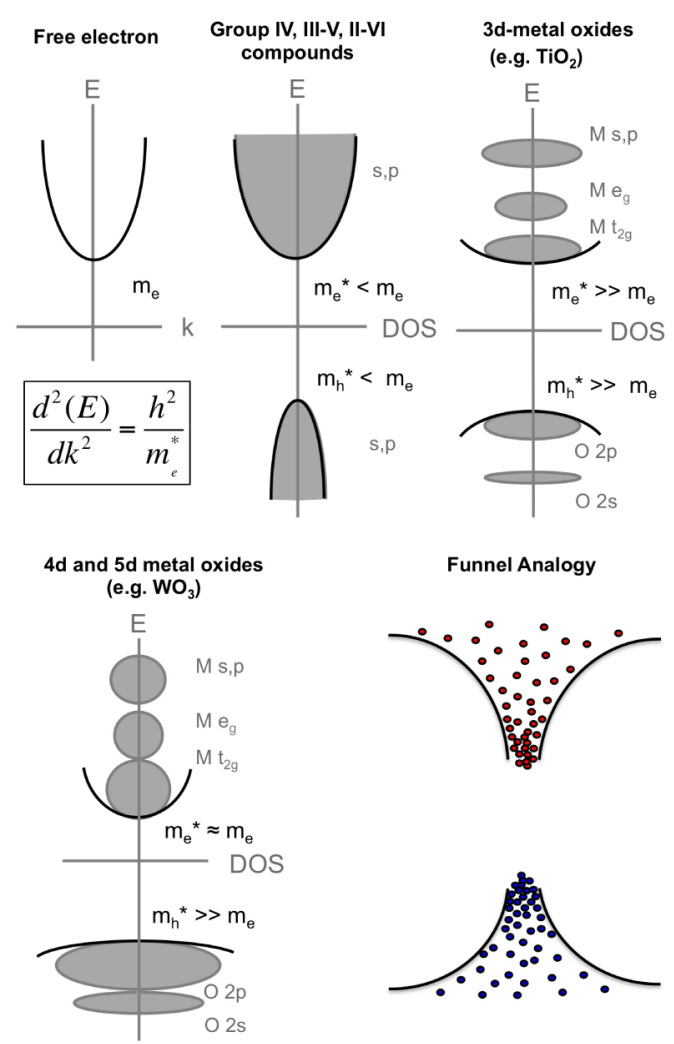

Figure 3. Energy-DOS plots for idealized semiconductors to illustrate relationships between composition, band curvature and effective masses for electrons and holes. The inertial effective mass tensor equation contains the wavevector $k$, the Plank constant $h$, and the electron effective mass, $m_{\mathrm{e}}{ }^{*}$ : High efficiency semiconductors have a "funnel-like" DOS, which concentrates charge carriers near the band edges.

parabola and the inertial effective mass $m^{*}$ corresponds to its inverse curvature. In a semiconductor lattice, the $E / k$ curve is modified by the allowed energy states, as defined by the number and arrangement of the atomic components, their orbitals, and their bonding interactions. ${ }^{14}$ Because in most crystal structures the packing of the atoms is anisotropic, the $E$ / $k$ curve and with it the effective mass are strongly directiondependent. To simplify this situation in semiconductors, the inertial effective mass tensor is replaced by the Density of States (DOS) Effective Mass as defined by eq 3.

$$
N_{\mathrm{CB} / \mathrm{VB}}=2\left(\frac{2 \pi m_{\mathrm{e} / \mathrm{h}}^{*} k T}{h^{2}}\right)^{3 / 2}
$$

Here the effective mass $m_{e}{ }^{*}$ is a function of the effective density of states $N_{\text {eff }}$ near the bottom of the energy-DOS curve. Steep bands with high curvature give small $N_{\text {eff }}$ and $m^{*}$ values, and small entropy, according to eq 2 . This situation is encountered among IV, III-V, and II-VI semiconductors $(\mathrm{Si}$, 
GaAs, CdTe) with a small number of orbitals per atom and high degree of covalency in all three dimensions.

On the other hand, flat bands with low curvature give large $N_{\text {eff }}$ and $m^{*}$ values, and large entropy, according to eq 2 . Such $E-D O S$ curves are expected for transition metal compounds due to the addition of five $\mathrm{d}$ orbitals per metal atom. In particular, for $3 \mathrm{~d}$ metal compounds, these orbitals have weak overlap and lead to degenerate states. Additionally, in multivalent metal compounds $\mathrm{MX}_{\mathrm{n}}$ flattening of the valence band is expected from adding $4 n(s+3 p)$ orbitals per formula unit. Examples are $\mathrm{TiO}_{2}(n=2)$ and $\mathrm{WO}_{3}(n=3)$. Slightly steeper E-DOS curves with intermediate $N_{\text {eff }}$ are expected for compounds of the heavier $4 \mathrm{~d}$ and $5 \mathrm{~d}$ metals, due to the stronger interactions among the spatially more extended $d$ orbitals. Qualitatively, the effect of the DOS on the free energy of the excited semiconductor can be expressed with the funnel analogy in Figure 3. The largest free energy per electron $\left(V_{\mathrm{OC}}\right)$ is available when the shape of the effective DOS resembles a funnel, that is, there is maximum concentration of charge carriers near the band edges. In contrast, a flat band structure will not concentrate charge carriers efficiently. On the other hand, further away from the band edges the DOS should be as large as possible, to maximize light absorption. This is because the absorption coefficient of a semiconductor is proportional to the DOS. Then the semiconductor harvests and concentrates the maximum amount of photons, like a funnel.

Using the effective mass concept, the entropy of the charge carriers in different semiconductors can be estimated with eq 2 . In Figure $4 \mathrm{~A}$ entropy values are plotted versus the effective mass (mean values of $m_{\mathrm{e}}{ }^{*}$ and $m_{\mathrm{h}}{ }^{*}$ from Table 1 ). Importantly, to allow direct comparison, the calculation assumes illumination conditions sufficient to generate a constant concentration of $10^{15} \mathrm{~cm}^{-3}$ charge carriers in each semiconductor. This is
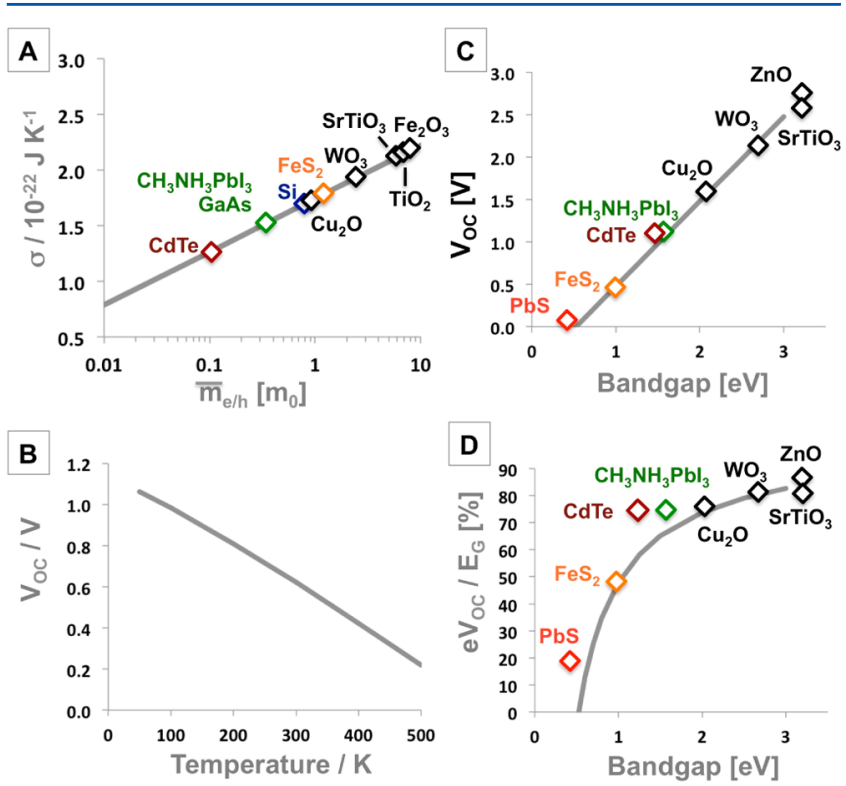

Figure 4. A. Entropy per charge carrier as a function of the mean effective mass. The calculation assumes $T=300 \mathrm{~K}$ and illumination sufficient to create $n_{\mathrm{e}}=n_{\mathrm{h}}=10^{15} \mathrm{~cm}^{-3}$. B. $V_{\text {OC }}$ dependence on temperature for Si-like semiconductor $\left(E_{\mathrm{G}}=1.12 \mathrm{eV}, \mathrm{m}_{\mathrm{e}}=1.08, \mathrm{~m}_{\mathrm{h}}=\right.$ $\left.0.55, n_{\mathrm{e}}=n_{\mathrm{h}}=10^{15} \mathrm{~cm}^{-3}\right)$. C. Dependence of $V_{\mathrm{OC}}$ on the bandgap $\left(300 \mathrm{~K}, \mathrm{~m}_{\mathrm{e}}=1.00\right.$ and $\left.\mathrm{m}_{\mathrm{h}}=1.00, n_{\mathrm{e}}=n_{\mathrm{h}}=10^{15} \mathrm{~cm}^{-3}\right)$. D. Same, but with $V_{\mathrm{OC}}$ as a fraction of the band gap energy. Individual data from Table 1. Deviations from the fit are due to changes in effective masses. different from fixed illumination (e.g., AM 1.5), where carrier concentrations would vary depending on the band gap of each material, and be subject to the principle of detailed balance. ${ }^{1,11,12}$ According to Figure 4A the entropy increases logarithmically with $m^{*}$, with minimal losses obtained for small values of $m^{*}$ (and correspondingly small $N_{\text {eff }}$ ). Such a situation is encountered for many group IV, III/V, and II/VI semiconductors $^{6}$ and also for the recently discovered lead halide perovskites. ${ }^{7,15-17}$ Thus, the reduced entropy in these materials can provide a simple explanation for the observed high efficiency of these compounds in photovoltaic devices (experimental data in Table 1). On the other hand, large entropy losses are expected for transition metal compounds, and especially oxides, due to their flat energy bands, and correspondingly, larger effective density of states. Indeed, no efficient transition metal oxide photovoltaic or photoelectrochemical devices have been found yet.

Knowing the entropy per charge carrier it is now possible to calculate the free energy of an illuminated semiconductor and its open circuit voltage. Equation 4 is obtained by summing the chemical potentials for electrons and holes (eq 1, note that $E_{\mathrm{CB}}$ for holes is negative) $)^{12}$ and by substituting the entropy terms with eqs 2 and 3 .

$$
\mu=E_{\mathrm{G}}-k T \ln \frac{N_{\mathrm{CB}} N_{\mathrm{VB}}}{n_{\mathrm{e}} n_{\mathrm{h}}}
$$

This is the well-known expression for the free energy of an electron hole pair as a function of the dilution of the charge carriers in the states near the band edges. ${ }^{13,18}$ High energy output is expected for semiconductors with small $N_{\text {eff }}$ which concentrate charge carriers near the band edges. Conversely, the more effective states, the greater the dilution and the lower the free energy and open circuit voltage of the device. Equation 4 can also be written as a function of the effective masses of electrons and holes (eq 5), which are tabulated for many semiconductors (Table 1). ${ }^{19}$

$$
\mu=E_{\mathrm{G}}-k T \ln \left(\frac{2 \pi k T}{h^{2}}\right)^{3} \frac{4\left(m_{\mathrm{e}}^{*} m_{\mathrm{h}}^{*}\right)^{3 / 2}}{n_{\mathrm{e}} n_{\mathrm{h}}}
$$

Equation 5 is useful for examining the effect of temperature on the open circuit voltage of an illuminated semiconductor (Figure 4B). The $V_{\mathrm{OC}} / T$ plot is a nearly straight line with a slope of $1.78 \mathrm{mV} \mathrm{K}^{-1}$. This is only slightly less than the experimentally observed temperature dependence of $\sim 2 \mathrm{mV}$ $\mathrm{K}^{-1}$ for actual silicon single junction devices under AM1.5 solar illumination. $^{20-22}$ The difference is partly due to the experimental increase of the bandgap with temperature, which is not considered in eq 5 .

Lastly, the effect of the semiconductor band gap on $V_{\mathrm{OC}}$ and on the ratio $V_{\mathrm{OC}} / E_{\mathrm{G}}$ (fraction of band gap energy that can be converted into usable energy) is plotted in Figure 4C and D. Naturally, at constant absorbed photon flux, any increase in band gap will lead to an increase in free energy $\left(\mathrm{e} V_{\mathrm{OC}}\right)$, due to the raised potential energy of the electron/hole pairs. As a result, the open circuit voltage varies linearly with band gap. However, $V_{\mathrm{OC}}$ goes to zero before the band gap does. This is because at ambient temperature the entropy term $\mathrm{T} \sigma$ outcompetes the potential and kinetic energy terms in eq 1 . Alternatively one can say that small band gaps increase $N_{\text {eff }}$, thereby diluting charge carriers and increasing the entropy $\sigma$. This limits the efficiency of small band gap semiconductors for solar energy conversion. This is a possible reason for the 
Table 1. Selected Semiconductor Parameters

\begin{tabular}{|c|c|c|c|c|c|c|c|c|c|c|c|c|}
\hline material & $\begin{array}{c}E_{\mathrm{G}} / \\
\mathrm{eV}\end{array}$ & $m^{*}{ }_{\mathrm{e}}$ & $m_{\mathrm{h}}^{*}$ & $\begin{array}{c}\text { mean } \\
m^{*}\end{array}$ & ref & $\sigma_{\mathrm{e}}^{a} / 10^{-22} \mathrm{~J}$ & $\sigma_{\mathrm{h}}^{a} / 10^{-22} \mathrm{~J}$ & $\begin{array}{c}V_{\mathrm{OC}} \\
(\text { calc })^{a}\end{array}$ & $\begin{array}{c}V_{\mathrm{OC}} / E_{\mathrm{G}} \\
(\%)^{a}\end{array}$ & $\begin{array}{c}V_{\mathrm{OC}} \\
(\exp ) / \mathrm{V}^{b}\end{array}$ & $\begin{array}{l}V_{\mathrm{OC}} / E_{\mathrm{G}_{b}}(\%) \\
\quad \exp .\end{array}$ & ref \\
\hline $\mathrm{sc}-\mathrm{Si}$ & 1.12 & 1.08 & 0.55 & 0.82 & 19 & 1.76 & 1.62 & 0.62 & 55 & 0.71 & 63 & 4 \\
\hline $\mathrm{CH}_{3} \mathrm{NH}_{3} \mathrm{PbI}_{3}$ & 1.55 & 0.23 & 0.29 & 0.26 & 23 & 1.44 & 1.49 & 1.13 & 73 & 0.99 & 64 & 4 \\
\hline InP & 1.28 & $\begin{array}{l}0.073 \\
(300 \mathrm{~K})\end{array}$ & $0.55(110 \mathrm{~K})$ & 0.31 & 19 & 1.20 & 1.62 & 0.88 & 69 & 0.88 & 69 & 4 \\
\hline $\mathrm{CdTe}$ & 1.45 & $0.096(1.8 \mathrm{~K})$ & $0.12(1.8 \mathrm{~K})$ & 0.11 & 19 & 1.26 & 1.30 & 1.10 & 76 & 0.86 & 59 & 4 \\
\hline GaAs & 1.42 & $0.067(1.6 \mathrm{~K})$ & 0.47 & 0.27 & 19 & 1.18 & 1.59 & 1.03 & 73 & 1.12 & 79 & 4 \\
\hline $\mathrm{Cu}_{2} \mathrm{O}$ & 2.1 & 0.99 & $0.58(1.7 \mathrm{~K})$ & 0.99 & 19 & 1.74 & 1.63 & 1.60 & 76 & 0.4 & 19 & 24 \\
\hline $\mathrm{ZnO}$ & 3.2 & $0.28(6 \mathrm{~K})$ & $0.59(1.6 \mathrm{~K})$ & 0.44 & 19 & 1.48 & 1.63 & 2.75 & 86 & - & - & \\
\hline $\mathrm{SrTiO}_{3}$ & 3.2 & 3 & 5 & 4 & 25 & 1.97 & 2.08 & 2.57 & 80 & $1.3^{c}$ & 41 & 26 \\
\hline $\mathrm{TiO}_{2}$ (anatase) & 3.1 & 10 & 0.8 & 5.4 & 27 & 2.22 & 1.70 & 2.50 & 81 & - & - & \\
\hline $\mathrm{WO}_{3}$ & 2.7 & 0.9 & 2 & 1.45 & 28 & 1.72 & 1.89 & 2.15 & 80 & & - & \\
\hline $\mathrm{Fe}_{2} \mathrm{O}_{3}$ & 2.06 & 13 & 2.1 & 7.55 & 29,30 & 2.27 & 1.90 & 1.41 & 68 & - & - & \\
\hline $\mathrm{FeS}_{2}$ & 1 & 0.44 & 1.61 & 1.02 & 31 & 1.57 & 1.84 & 0.49 & 49 & 0.5 & 0.5 & 32 \\
\hline $\mathrm{PbS}$ & 0.42 & $\begin{array}{c}0.08-0.11 \\
(4 \mathrm{~K})\end{array}$ & $\begin{array}{c}0.08-0.11 \\
(4 \mathrm{~K})\end{array}$ & 0.09 & 19 & 1.26 & 1.26 & 0.08 & 19 & - & - & \\
\hline
\end{tabular}

${ }^{a}$ At $300 \mathrm{~K}$ for $10^{15}$ carriers $\mathrm{cm}^{-3}$; ${ }^{b}$ At AM $1.5 ;{ }^{c} \lambda<400 \mathrm{~nm}$.

current lack of efficient bulk PbS $\left(E_{\mathrm{G}}=0.42 \mathrm{eV}\right)$ or PbSe $\left(E_{\mathrm{G}}=\right.$ $0.27 \mathrm{eV})$ solar cells. ${ }^{19}$ Better efficiency with such semiconductors could be achieved theoretically by reducing their temperature (Figure 4B) with passive cooling (wind, water), because lower temperature reduces $N_{\text {eff }}$.

Using the insights from the entropy model, the power output from an excited semiconductor can be improved theoretically by increasing the steepness of the $E / k$ parabola. In consequence both $\mathrm{N}_{\mathrm{CB} / \mathrm{VB}}$ and $\sigma$ will decrease, according to eq 4 . Such a reshaping of the DOS structure can readily be achieved in small nanocrystals using the quantum size effect (Figure 5). Here, for

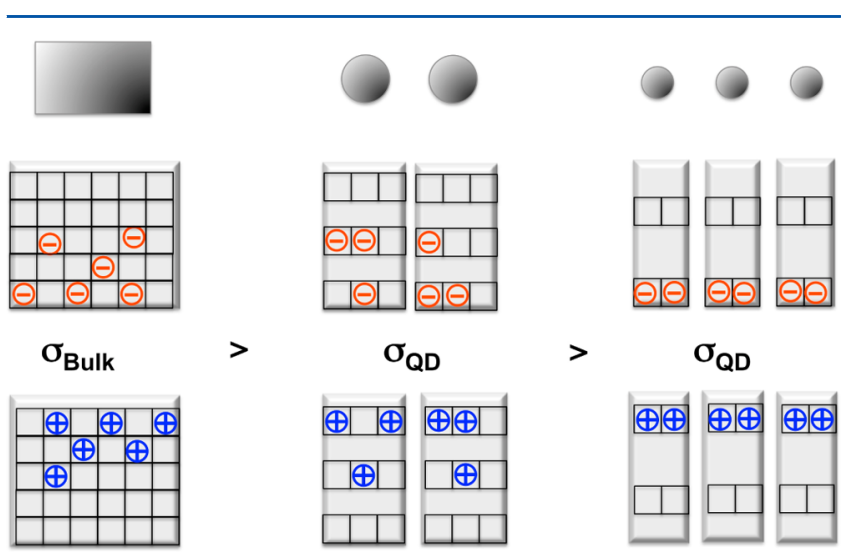

Figure 5. Effect of quantum sizing on $N_{\text {eff }}$ and entropy for three systems of $6 \mathrm{e} / \mathrm{h}$ pairs.

$N_{\text {eff }}=18,12,6$, and $n=6$ electrons, $W_{\text {tot }}=18 ! /[(18-6) ! 6 !]$, $12 ! /[(12-6) ! 6 !], 6 ! /[(6-6) ! 6 !]$, and $\sigma=1.5 \times 10^{-23}, 1.1 \times$ $10^{-23}, 0.0 \mathrm{~J} / \mathrm{K}$. Thus, the free energy increases with quantum confinement due to the reduction of the effective DOS near the band edges. Physically, this represents a concentration of charge carriers near the $\mathrm{CB}$ and $\mathrm{VB}$ edges.

To achieve sufficient charge concentration, the energy separation between allowed states should be several times $k T$. This behavior is often found in molecular light absorbers, because both high covalency and small number of atoms favor a vertical arrangement of energy states. Chlorophyll is a good example for this behavior. ${ }^{33}$ Because of the $0.73 \mathrm{eV}(30 \mathrm{kT})$ energy separation between excited states, only the first exited state is occupied at room temperature. Thus, the excited state entropy is near zero and free energy is at maximum. This situation formally corresponds to effective masses $m_{\mathrm{e} / \mathrm{h}}<1$, as shown in Figure 4A. The bipyridyl $\mathrm{Ru}$ dyes in dye sensitized photoelectrochemical cells are a second example. Here, the energy levels are separated by $0.3 \mathrm{eV}(>10 \mathrm{kT}$ at $300 \mathrm{~K}){ }^{34}$ This may be one of the reasons for the $>10 \%$ energy efficiency of molecular dye sensitized photovoltaic cells. ${ }^{35}$ But the lower DOS in molecular solar energy conversion systems also have an unwanted side effect: It reduces the light absorption coefficient, which is proportional to DOS. Nature has solved this problem by surrounding the chlorophyll absorber with an antenna system that absorbs the light and funnels it to the reaction site. ${ }^{36}$ This preconcentration of light ensures efficient excitation of the reaction site, even under low illumination conditions. Thus, it appears that in the presence of a light concentrating mechanism, molecular and quantum confined systems are better suited for photochemical energy conversion.

For devices with small light absorbers (molecules, quantum dots) one also has to consider that the energetics of such systems are very sensitive to variations in size, shape, and molecular environment. Because of this effect, photovoltaic cells with polydisperse and randomly packed quantum dots are expected to have a larger ground state entropy than those consisting of monodisperse and ordered dots. If the individual units are coupled electronically, the free energy of the ensemble will decrease, by redistribution of charge among the absorbers. This exactly reverses the entropy reduction in the individual subsystems. Indeed, material disorder in photovoltaic cells has been identified as a detriment to high power conversion efficiency. ${ }^{37}$ This effect could also be responsible for the observed $V_{\mathrm{OC}}$ losses in Ternary Blend Bulk Heterojunction Organic Solar Cells. ${ }^{38}$

In theory, entropy losses in such devices can be minimized by electronically separating light absorbers from each other. This would prevent the entropy increase from the free energy equilibrium between each absorber. Alternatively, all light absorbers in the ensemble should be made identical in size, shape, and chemical environment, thereby eliminating the effect of ground state entropy. Nature is already applying both design principles for minimization of excited state entropy. In the photosynthetic membrane the reactive centers are identical in structure and sufficiently isolated to prevent the free energy equilibrium between the charge carriers in them. One can 
expect that by adopting these design principles artificial light conversion devices could also achieve higher efficiency.

In summary, we have shown that the extractable maximum work from photovoltaic and photocatalytic devices does not just depend on the semiconductor band gap, but also on the excited state entropy. This entropy loss can be estimated with the modified Sackur Tetrode equation as a function of the curvature of the bands, the degeneracy of states near the band edges, the illumination intensity, the temperature, and the band gap. The application of the second law to semiconductors provides a simple explanation for the observed high performance in semiconductors with tetrahedral bonding in the zincblende structure types and for the lower efficiency of metal chalcogenides and oxides containing weakly interacting metal d orbitals. The entropy model also predicts low entropy losses in quantum confined and molecular structures, due to lower degeneracy of excited states.

\section{AUTHOR INFORMATION}

\section{Corresponding Author}

*E-mail: fosterloh@ucdavis.edu.

\section{Notes}

The authors declare no competing financial interest.

\section{ACKNOWLEDGMENTS}

F.E.O. is grateful for financial support from Research Corporation for Science Advancement (Scialog award) and from the National Science Foundation (NSF, Grants 1152250 and 1133099).

\section{REFERENCES}

(1) Shockley, W.; Queisser, H. J. Detailed Balance Limit Of Efficiency Of P-N Junction Solar Cells. J. Appl. Phys. 1961, 32, 510519.

(2) Ross, R. T.; Hsiao, T. L. Limits On Yield Of Photochemical Solar-Energy Conversion. J. Appl. Phys. 1977, 48, 4783-4785.

(3) Ross, R. T. Some Thermodynamics Of Photochemical Systems. J. Chem. Phys. 1967, 46, 4590-\&.

(4) Nayak, P. K.; Bisquert, J.; Cahen, D. Assessing Possibilities and Limits for Solar Cells. Adv. Mater. 2011, 23, 2870-2876.

(5) Nayak, P. K.; Cahen, D. Updated Assessment of Possibilities and Limits for Solar Cells. Adv. Mater. 2014, 26, 1622-1628.

(6) Green, M. A.; Emery, K.; Hishikawa, Y.; Warta, W.; Dunlop, E. D. Solar Cell Efficiency Tables (Version 41). Prog. Photovolt. 2013, 21 (1), 1-11.

(7) Kojima, A.; Teshima, K.; Shirai, Y.; Miyasaka, T. Organometal Halide Perovskites as Visible-Light Sensitizers for Photovoltaic Cells. J. Am. Chem. Soc. 2009, 131, 6050-6051.

(8) Huda, M. N.; Al-Jassim, M. M.; Turner, J. A. Mott Insulators: An Early Selection Criterion For Materials For Photoelectrochemical $\mathrm{H}(2)$ Production. J. Renewable Sustainable Energy 2011, 3, 053101-1053101-10.

(9) Krol, R., Principles of Photoelectrochemical Cells. In Photoelectrochemical Hydrogen Production; van de Krol, R., Grätzel, M., Eds.; Springer: New York, 2012; Vol. 102, pp 13-67.

(10) Cox, P. A. Transition Metal Oxides: An Introduction To Their Electronic Structure And Properties; Clarendon Press/Oxford University Press: Oxford/New York, 2010.

(11) Ruppel, W.; Wurfel, P. Upper Limit For The Conversion Of Solar-Energy. IEEE Trans. Electron Devices 1980, 27, 877-882.

(12) Würfel, P. Physics of Solar Cells; Wiley-VCH: Weinheim, 2005; p 244.

(13) Salvador, P. Semiconductors' Photoelectrochemistry: A Kinetic and Thermodynamic Analysis in the Light of Equilibrium and Nonequilibrium Models. J. Phys. Chem. B 2001, 105, 6128-6141.
(14) Hoffmann, R. How Chemistry and Physics Meet in the SolidState. Angew. Chem., Int. Ed. Engl. 1987, 26, 846-878.

(15) Burschka, J.; Pellet, N.; Moon, S. J.; Humphry-Baker, R.; Gao, P.; Nazeeruddin, M. K.; Gratzel, M. Sequential Deposition As A Route To High-Performance Perovskite-Sensitized Solar Cells. Nature 2013, 499, 316-320.

(16) Lee, M. M.; Teuscher, J.; Miyasaka, T.; Murakami, T. N.; Snaith, H. J. Efficient Hybrid Solar Cells Based on Meso-Superstructured Organometal Halide Perovskites. Science 2012, 338, 643-647.

(17) Manser, J. S.; Kamat, P. V. Band Filling With Free Charge Carriers In Organometal Halide Perovskites. Nat. Photonics 2014, 8, 737-743.

(18) Gerischer, H. Stability Of Semiconductor Electrodes Against Photodecomposition. J. Electroanal. Chem. 1977, 82, 133-143.

(19) Madelung, O. Semiconductors: Data Handbook, 3rd ed.; Springer: Berlin, 2004; p 691 p.

(20) Wu, C. Y.; Chen, J. F. Temperature Coefficients Of The OpenCircuit Voltage Of P-N-Junction Solar-Cells. J. Appl. Phys. 1982, 53, $3852-3858$.

(21) Hall, R. N. Silicon Photo-Voltaic Cells. Solid-State Electron. 1981, 24, 595-616.

(22) Khoury, A.; Charles, J. P.; Charette, J.; Fieux, M.; Mialhe, P. Solar-Cells - A Laboratory Experiment On The TemperatureDependence Of The Open-Circuit Voltage. Am. J. Phys. 1984, 52, 449-451.

(23) Du, M. H. Efficient Carrier Transport In Halide Perovskites: Theoretical Perspectives. J. Mater. Chem. A 2014, 2, 9091-9098.

(24) Paracchino, A.; Laporte, V.; Sivula, K.; Graetzel, M.; Thimsen, E. Highly Active Oxide Photocathode for Photoelectrochemical Water Reduction. Nat. Mater. 2011, 10, 456-461.

(25) Wunderlich, W.; Ohta, H.; Koumoto, K. Enhanced Effective Mass in Doped $\mathrm{SrTiO}_{3}$ and Related Perovskites. Physica B 2009, 404, 2202-2212.

(26) Wrighton, M. S.; Ellis, A. B.; Wolczanski, P. T.; Morse, D. L.; Abrahamson, H. B.; Ginley, D. S. Strontium-Titanate Photoelectrodes - Efficient Photoassisted Electrolysis of Water at Zero Applied Potential. J. Am. Chem. Soc. 1976, 98, 2774-2779.

(27) Enright, B.; Fitzmaurice, D. Spectroscopic Determination of Electron And Hole Effective Masses in a Nanocrystalline Semiconductor Film. J. Phys. Chem. 1996, 100, 1027-1035.

(28) Berak, J. M.; Sienko, M. J. Effect of Oxygen-Deficiency on Electrical Transport Properties of Tungsten Trioxide Crystals. J. Solid State Chem. 1970, 2, 109-133.

(29) Shaner, M. R.; Fountaine, K. T.; Ardo, S.; Coridan, R. H.; Atwater, H. A.; Lewis, N. S. Photoelectrochemistry of Core-Shell Tandem junction n-p+-Si/n-WO3Microwire Array Photoelectrodes. Energy Environ. Sci. 2014, 7, 779-790.

(30) Peng, H. W.; Lany, S., Semiconducting Transition-Metal Oxides Based on $\mathrm{d}(5)$ Cations: Theory for $\mathrm{MnO}$ and $\mathrm{Fe}_{2} \mathrm{O}_{3}$. Phys. Rev. B 2012, 85 .

(31) Hu, J.; Zhang, Y. N.; Law, M.; Wu, R. Q. Increasing the Band Gap of Iron Pyrite by Alloying with Oxygen. J. Am. Chem. Soc. 2012, 134, 13216-13219.

(32) Ennaoui, A.; Fiechter, S.; Pettenkofer, C.; Alonsovante, N.; Buker, K.; Bronold, M.; Hopfner, C.; Tributsch, H. Iron Disulfide For Solar-Energy Conversion. Sol. Energy Mater. Sol. C 1993, 29, 289-370.

(33) Linnanto, J.; Korppi-Tommola, J. Quantum Chemical simulation of Excited States of Chlorophylls, Bacteriochlorophylls and Their Complexes. Phys. Chem. Chem. Phys. 2006, 8, 663-687.

(34) Nazeeruddin, M. K.; De Angelis, F.; Fantacci, S.; Selloni, A.; Viscardi, G.; Liska, P.; Ito, S.; Bessho, T.; Gratzel, M. Combined Experimental and DFT-TDDFT Computational Study of Photoelectrochemical Cell Ruthenium Sensitizers. J. Am. Chem. Soc. 2005, $127,16835-16847$.

(35) Hagfeldt, A.; Boschloo, G.; Sun, L. C.; Kloo, L.; Pettersson, H. Dye-Sensitized Solar Cells. Chem. Rev. 2010, 110, 6595-6663.

(36) Scholes, G. D.; Fleming, G. R.; Olaya-Castro, A.; van Grondelle, R. Lessons From Nature About Solar Light Harvesting. Nat. Chem. 2011, 3, 763-774. 
(37) Nayak, P. K.; Garcia-Belmonte, G.; Kahn, A.; Bisquert, J.; Cahen, D. Photovoltaic Efficiency Limits and Material Disorder. Energy Environ. Sci. 2012, 5, 6022-6039.

(38) Street, R. A.; Davies, D.; Khlyabich, P. P.; Burkhart, B.; Thompson, B. C. Origin of the Tunable Open-Circuit Voltage in Ternary Blend Bulk Heterojunction Organic Solar Cells. J. Am. Chem. Soc. 2013, 135, 986-989. 